\title{
Low-noise, GHz repetition-rate femtosecond lasers and frequency combs: implementation and applications
}

\author{
Franz X. Kärtner ${ }^{1,2,3}$ and Guoqing Chang ${ }^{1,3}$ \\ ${ }^{1}$ Center for Free-Electron Laser Science, DESY, Notkestraße 85, 22607 Hamburg, Germany \\ ${ }^{2}$ Physics Department, University of Hamburg, Luruper Chaussee 149, 22761 Hamburg, Germany \\ ${ }^{3}$ Department of Electrical Engineering and Computer Science and Research Laboratory of Electronics, \\ Massachusetts Institute of Technology, 77 Massachusetts Avenue, Cambridge, MA 02139, USA
}

\section{(Invited Paper)}

\begin{abstract}
We report on advances and unique characteristics of high repetition-rate $(>1 \mathrm{GHz})$ Ti:Sapphire, Er-fiber, and Yb-fiber femtosecond laser frequency combs, and their applications in high-speed, high resolution optical sampling and astrophysical spectrograph calibration.

(C) 2013 Optical Society of America

OCIS codes: (140.4050) Mode locked lasers; (320.7085) Ultrafast information processing; (120.620) Spectrometers and spectroscopic instrumentation
\end{abstract}

\section{Introduction}

Femtosecond mode-locked lasers exhibiting ultralow noise have opened new research possibilities. Measurement of phase noise in optical pulse trains by converting to a radio frequency signal is extremely difficult because RF measurement techniques are limited by thermal noise in terminating resistors as well as the signal level available for input to the phase noise measurement system. Recently we have demonstrated a direct optical technique - balanced optical cross correlation - with higher sensitivity by avoiding the photo-detection process [1]. We measured the timing error spectrum between phase locked optical pulse trains produced from two nearly identical 10-fs Ti:Sapphire lasers running at $82 \mathrm{MHz}$, and demonstrated a record low integrated timing error of less than 13 attoseconds (measured from DC to the Nyquist frequency of the pulse train) [2]. This corresponds to the lowest value of high frequency phase noise ever recorded of $-203 \mathrm{dBc} / \mathrm{Hz}$ (assuming a $10 \mathrm{GHz}$ carrier) for offset frequencies greater than $1 \mathrm{MHz}$. For many applications, high repetition-rate $(>1 \mathrm{GHz})$ lasers with femtosecond pulse duration are required in addition to ultralow noise. For nonlinear bio-optical imaging in which photo-induced damage is caused by pulse energy rather than average power, increasing pulse repetition-rate will improve signal-tonoise ratio and reduce data acquisition time. Frequency combs - achieved by fully stabilizing both the repetitionrate and the carrier-envelope offset frequency of multi-GHz lasers - exhibit large line spacing that may permit access to and manipulation of each individual comb line. Such capabilities have opened numerous frequencydomain applications including optical arbitrary waveform generation, high-speed analog-to-digital conversion, and high-resolution spectroscopy.

2. GHz Ti:sapphire laser frequency comb for astrophysical spectrograph calibration
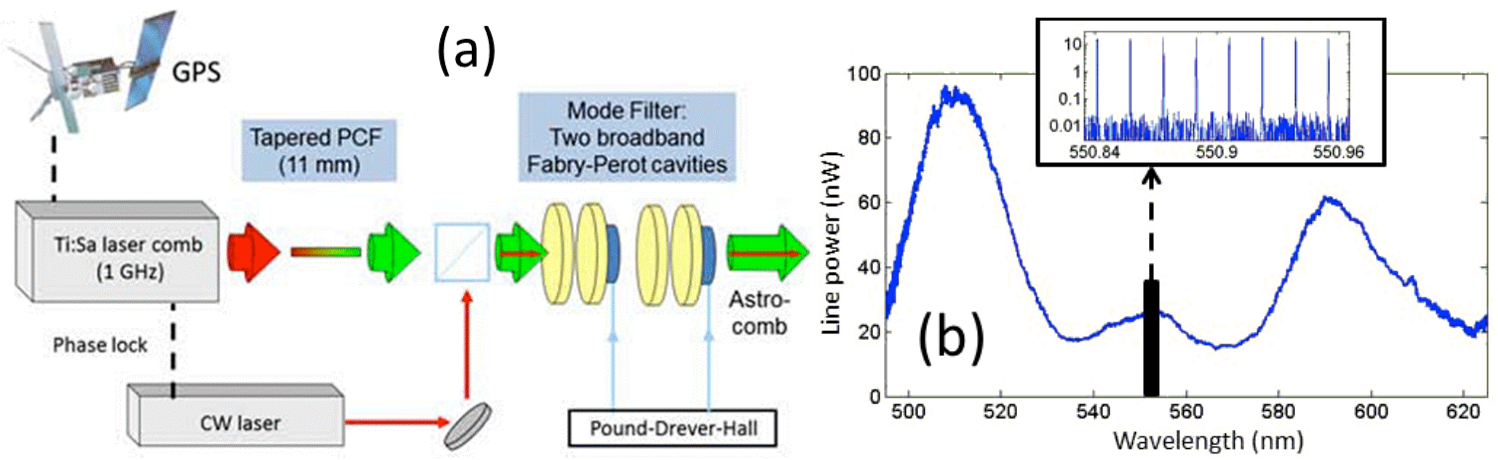

Fig. 1 (a) The block diagram of our green astro-comb setup. (b) The green astro-comb spectrum. Our green astro-comb is composed of $>7000$ ultra-accurate lines equally spaced by $16 \mathrm{GHz}$ from $500-620 \mathrm{~nm}$. The broadband spectrum shown here is measured by a commercial optical spectrum analyzer and the zoom-in spectrum (insert) is measured by a high resolution Fourier transform interferometer built in house.

By stabilizing the repetition rate and carrier-envelope offset frequency of an octave-spanning 1-GHz Ti:sapphire laser, the resulted 1-GHz laser frequency comb has enabled the key device ("astro-comb") to calibrate astrophysical spectrographs for Earth-like planets detection [3]. After referencing the comb to an atomic clock locked to the 
Global Positioning System (GPS), the frequency of each astro-comb line is accurate to a fractional uncertainty of $10^{-}$ 12. To transfer the astro-comb accuracy to the stellar radial-velocity measurements using an astrophysical spectrograph, an astro-comb must have 1) many (>2000) widely spaced ( $>10 \mathrm{GHz})$ spectral lines resolvable by the spectrograph, 2) homogenous line power, and 3) broad wavelength coverage over the stellar spectral bands with many sharp and stable features (typically $400-600 \mathrm{~nm}$ ). We have developed a green astro-comb (Fig. 1(a)) and deployed it at the Telescopio Nazionale Galileo (TNG) on La Palma in the Canary Islands as a calibration source for the HARPS-N spectrograph, which is performing precision radial-velocity observations of bright stars to detect and characterize long-period, Earth-like exoplanets. The spectrum of our green astro-comb is shown in Fig. 1(b). In the spectral band of $500-620 \mathrm{~nm}$, there are $>7000$ astro-comb lines equally spaced by $16 \mathrm{GHz}$ (optimized for HARPSN) with powers varying by less than $8 \mathrm{~dB}$. The frequency of each astro-comb line is accurate to $1 \mathrm{~cm} / \mathrm{s}$ level over years because it is referenced to GPS.

\section{GHz Er-fiber mode-locked laser for high-speed sampling}

Accurate conversion of wideband multi-GHz analog signals into the digital domain has long been a target of analogto-digital converter (ADC) developers, driven by applications in radar systems, software radio, medical imaging, and communication systems. Aperture jitter has been a major bottleneck on the way towards higher speeds and better accuracy. Photonic ADCs, which perform sampling using ultra-stable optical pulse trains generated by modelocked lasers, are a promising approach to overcome the jitter problem and bring ADC performance to new levels. Recently we have developed an ultra-low-jitter, 1-GHz Er-fiber mode-locked laser; it has enabled us to demonstrate that the photonic approach can deliver on its promise by digitizing a $41 \mathrm{GHz}$ signal with 7.0 effective bits using a photonic ADC built from discrete components; see Fig. 2 [4]. This accuracy corresponds to a timing jitter of $15 \mathrm{fs}-$ a 4-5 times improvement over the performance of the best electronic ADCs which exist today. On the way towards an integrated photonic ADC, a silicon photonic chip with core photonic components was fabricated and used to digitize a $10 \mathrm{GHz}$ signal with 3.5 effective bits. In these experiments, two wavelength channels were implemented, providing the overall sampling rate of $2.1 \mathrm{GSa} / \mathrm{s}$. To show that photonic ADCs with larger channel counts are possible, a dual 20-channel silicon filter bank has been demonstrated.

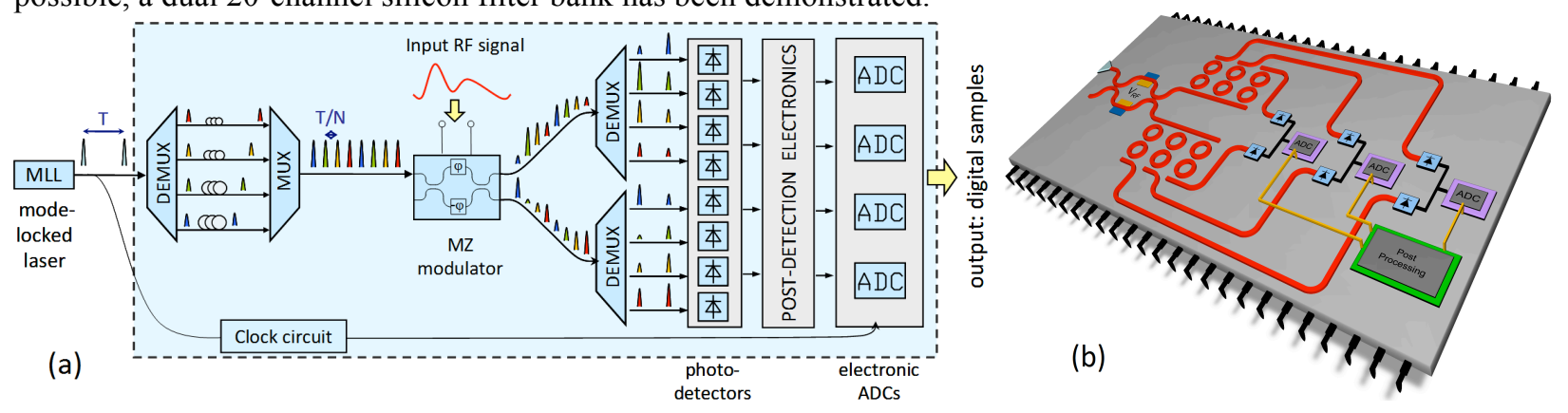

Fig 2. (a) Layout of the photonic ADC studied here; the components inside the dashed box can in principle be integrated on a single chip; (b) a vision for the photonic $\mathrm{ADC}$ implemented as a single electronic-photonic silicon chip.

\section{3-GHz Yb-fiber mode-locked laser: filling spectral gap}

For many other applications, $\mathrm{GHz}$ femtosecond pulses are required at the wavelength range that cannot be covered by Ti:sapphire lasers (centered at $800 \mathrm{~nm}$ ) and Er-fiber lasers (centered at $1.55 \mu \mathrm{m}$ ). For example, most live biological specimens exhibit a minimum light attenuation in the range of 1.2-1.35 $\mu \mathrm{m}$. Using GHz femtosecond pulses of this wavelength range for nonlinear bio-optical imaging allows deeper penetration through turbid specimens and meanwhile avoids photo-induced damage caused by pulse energy. Recently we have demonstrated a 3-GHz Yb-fiber laser system with $>10-\mathrm{W}$ average power, constituting a powerful laser platform to access other wavelength range via nonlinear wavelength conversion [5]. Using stimulated Raman scattering inside photonic crystal fibers, we have implemented a 3-GHz femtosecond Raman soliton source tunable between 1.06-1.35 $\mu \mathrm{m}$; at $1.35 \mu \mathrm{m}$, the source produces average power of $900 \mathrm{~mW}$; using fiber-optic Cherenkov radiation, we have demonstrated a $3-\mathrm{GHz}, 11-\mathrm{fs}$ source centered at $850 \mathrm{~nm}$ with $>300-\mathrm{mW}$ average power-a potential substitute of multi-GHz Ti:sapphire lasers.

\section{References}

[1] Kim et al, "Attosecond-precision ultrafast photonics," Laser \& Photonics Reviews 4, p. 432 (2010).

[2] Benedick et al., "Optical flywheels with attosecond jitter," Nature Photonics 6, 97 (2012).

[3] C.-H. Li et al., "A laser frequency comb that enables radial velocity measurements with a precision of $1 \mathrm{~cm} / \mathrm{s}$," Nature 452 , 610 (2008).

[4] Khilo et al., "Photonic ADC: overcoming the bottleneck of electronic jitter," Opt. Express 20, 4454 (2012).

[5] H. -W. Chen et al., "3 GHz, fundamentally mode-locked, femtosecond Yb-fiber laser," Opt. Lett. 37, 3522 (2012). 